\title{
Lymphoid interstitial pneumonitis associated with common variable hypogammaglobulinaemia treated with cyclosporin A
}

\author{
Christopher W H Davies, Mark C Juniper, Winifred Gray, Fergus V Gleeson, \\ Helen M Chapel, Robert J O Davies
}

\begin{abstract}
Lymphoid interstitial pneumonitis (LIP) is a rare clinicopathological entity that may be associated with common variable immune deficiency (CVID) and may lead to respiratory failure and death. Some patients may respond to prolonged corticosteroid treatment. We hypothesised that, in view of the predominant $T$ cell nature of LIP, cyclosporin $A$ would be a more appropriate choice of immunosuppressive agent and report the first case of its successful use in a woman with LIP associated with CVID.

(Thorax 2000;55:88-90)
\end{abstract}

Keywords: lymphoid interstitial pneumonitis; common variable immune deficiency; cyclosporin A

Lymphoid interstitial pneumonitis (LIP) is a rare clinicopathological entity that is characterised by a diffuse or multifocal reactive lymphoid infiltrate involving the alveolar interstitium. ${ }^{1-4}$ Most cases show a preponderance of lymphocytes. ${ }^{1-5}$ The condition has been associated with a number of diverse systemic illnesses such as AIDS, Epstein-Barr virus infection, autoimmune disorders, and various

Osler Chest Unit, Churchill Hospital, Oxford Radcliffe Trust, Oxford OX3 7LJ, UK C W H Davies M C Juniper R J O Davies

Department of Radiology F V Gleeson

Department of Cellular Pathology, John Radcliffe Hospital, Oxford Radcliffe Trust, Oxford OX3 9DU, UK

W Gray

Department of Immunology H M Chapel

Correspondence to: Dr CWH Davies

Received 9 October 1997 Returned to authors 21 January 1998 Revised manuscript received 25 February 1998

Accepted for publication 16 March 1998 immunodeficiency states including common variable immune deficiency (CVID), also known as primary antibody deficiency. ${ }^{1-3}$

The association of LIP with CVID was first reported by Liebow and Carrington in $1973^{6}$ and up to $10 \%$ of patients with LIP may have hypogammaglobulinaemia. ${ }^{13}$ The clinical course of non-AIDS LIP is variable but death may occur due to progressive pulmonary fibrosis though some patients may respond to corticosteroid therapy taken for prolonged periods. ${ }^{2-5}$ There are only occasional reports of treatment with alternative immunosuppressants. ${ }^{27}$ We hypothesised that, in view of the predominant $\mathrm{T}$ cell nature of LIP, cyclosporin A would be a more appropriate choice of immunosuppressive agent and report the first case of its successful use in a patient with LIP associated with CVID.

\section{Case report}

In October 1995 a 34 year old woman with known CVID diagnosed in 1989 was admitted to our unit complaining of dyspnoea and fatigue for two months. She had been treated with intravenous immunoglobulin replacement therapy since 1989 at a dose of $0.3 \mathrm{~g} / \mathrm{kg} / \mathrm{month}$ in divided doses. She had previously been diagnosed as having bronchiectasis which was treated with long term antibiotics, and autoimmune haemolytic anaemia which was treated with corticosteroids and splenectomy. As a result of steroid treatment she had developed severe osteoporosis with multiple previous pathological bone fractures.

On admission she complained of fever, sweats, and progressive dyspnoea with nocturnal cough increasing over the preceding two weeks. She had been given several courses of oral antibiotics and inhaled budesonide with no improvement. She had never smoked. On examination she was apyrexial with bilateral crackles on auscultation and a splenectomy scar.

Initial investigations showed leucocytosis at $12.9 \times 10^{9} / 1$ (neutrophils $6.5 \times 10^{9} / 1$, total lymphocytes $4.2 \times 10^{9} / 1$, T cells $2.5 \times 10^{9} / 1, \mathrm{CD} 4$ lymphocytes $1.82 \times 10^{9} / 1$, CD8 lymphocytes $0.68 \times 10^{9} / 1$ ) and $\mathrm{C}$ reactive protein concentrations of $36 \mathrm{mg} / \mathrm{l}$. Chest radiography and high resolution computed tomography (HRCT) showed patchy ground glass opacification, peribronchovascular nodules, discrete nodules, and areas of bronchiectasis. Antinuclear antibodies, Aspergillus precipitins, and blood cultures were negative. Her immunoglobulin profile at admission after recent infusion of immunoglobulin (normal ranges) was IgG 14 (6-13) g/l, IgA <0.1 (0.8-3.9) g/1, IgM 1.6 $(0.4-2.5) \mathrm{g} / \mathrm{l}$, and IgE $4(<120) \mathrm{kU} / 1$. Pulmonary function showed a restrictive pattern (forced expiratory volume in one second $\left(\mathrm{FEV}_{1}\right) \quad 1.721$ (58\% predicted), forced vital capacity (FVC) 1.901 (56\% predicted) with reduced gas transfer coefficient (KCO) $62 \%$ predicted).

Immediate management included broad spectrum antibiotics but, after no improvement and continued fever, bronchoscopic lavage was performed; this did not identify any bacteria, mycobacteria, virus or fungi on culture. Polymerase chain reaction (PCR) for DNA to Epstein-Barr virus, cytomegalovirus, and Mycoplasma pneumoniae was negative. Ten days after admission the patient underwent an open lung biopsy (lingula) for diagnosis.

Histological examination showed an extensive lymphocytic infiltrate of the lung parenchyma with peribronchial germinal centre formation and epithelialisation of some alveoli (fig 1). Immunohistochemistry showed the lymphocytes to be predominantly $\mathrm{T}$ cells with only sparse representation of B cells. Most of the T cells were CD4 positive with only a few CD8 


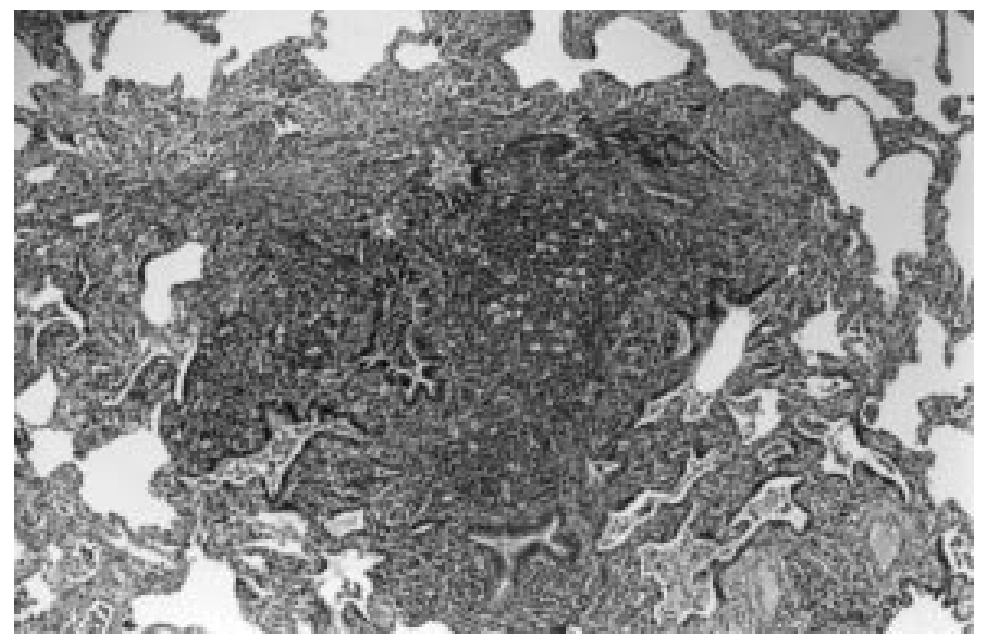

Figure 1 Lymphocytic infiltration of alveolar walls with peribronchiolar lymphoid follicles and germinal centre formation. Epithelialised alveoli are also present. Stain: $H \mathcal{E} E(\times$ medium power). ated with bilateral new pulmonary nodules and increased ground glass opacification on the HRCT chest scan. She remained on prednisolone in a dose of $7.5 \mathrm{mg}$ on alternate days and the dose of cyclosporin was increased to $125 \mathrm{mg} /$ day with a subsequent improvement in clinical symptoms and lung function $\left(\mathrm{FEV}_{1}\right.$ 2.321 ; FVC 2.611 ; KCO 93\% predicted). There was associated resolution of the chest radiograph and CT scan infiltrates and nodules. Currently the patient is in remission on prednisolone in a dose of $7.5 \mathrm{mg}$ on alternate days with a serum cyclosporin level of $76 \mathrm{ng} /$ $\mathrm{ml}$.

\section{Discussion}

Lymphoid interstitial pneumonitis (LIP) is by definition a reactive lymphoid infiltrate involving the pulmonary interstitium ${ }^{1-4}$ and is also known as diffuse hyperplasia of the bronchus associated lymphoid tissue (BALT). ${ }^{3}$ BALT is thought to be an important factor in the immunological response to inhaled antigens landing on the mucosal surfaces and is part of the more widely recognised mucosa associated immune system. The entity of LIP may be seen in association with a variety of conditions such as paediatric AIDS, primary immunodeficiency, Sjögren's syndrome, myasthenia gravis, chronic liver disease, and dysproteinaemic states including hyper- and hypogammaglobulinaemia. ${ }^{1-4} \mathrm{~A}$ small number of cases may in fact be a diffuse interstitial low grade $\mathrm{B}$ cell lymphoma with microscopic foci resembling LIP. ${ }^{1} \mathrm{Up}$ to $80 \%$ of patients with LIP will have evidence of dysproteinaemia, most frequently hypergammaglobulinaemia due to elevation of $\operatorname{IgM}$ and IgG. ${ }^{3}$ However, hypogammaglobulinaemia occurs in about $10 \%$ of adults with this condition, and the association of LIP with CVID has been documented for over 20 years. ${ }^{13}$

Histologically, LIP associated with hypogammaglobulinaemia is characterised by infiltration of the alveolar interstitium by $\mathrm{T}$ lymphocytes and also plasma cells, fibroblasts, and scattered macrophages. ${ }^{1-4}$ In non-AIDS patients the lymphoid infiltrate is centred on the airways but invariably infiltrates the interstitium and lymphoid follicles, usually peribronchiolar, are present in many cases. ${ }^{1}$ The pattern is similar to that seen in reactive lymph nodes, suggesting that the lung is acting like a giant lymphoid organ. In cases of LIP associated with CVID the cells consist largely of $\mathrm{T}$ cells, particularly CD4+ T helper cells. ${ }^{1}$ It is important to exclude monoclonality in the cell populations which would favour an alternative diagnosis of lymphoma, also a recognised association with CVID. ${ }^{35}$

CVID is primarily a defect in the function of B lymphocytes, usually characterised by the presence of low or normal numbers of B cells which fail to mature into plasma cells when exposed to antigens or mitogens. ${ }^{8}$ Most patients have normal $\mathrm{T}$ lymphocyte CD4:CD8 ratios, although a rare subgroup with CVID have a reduced ratio with a lymphopenia primarily due to CD4+CD45 RA+(unprimed) $\mathrm{T}$ cells suggesting persistent activation. ${ }^{8}$ 
The optimal treatment for LIP is not well established and, due to the small number of cases, there are no controlled trials. However, most patients are treated by prolonged courses of corticosteroids which may control the process or induce remission, ${ }^{2-5}$ and a few reports have described the additional use of the immunosuppressive agents methotrexate, ${ }^{2}$ azathioprine, ${ }^{2}$ and chlorambucil ${ }^{7}$ in conjunction with corticosteroids.

The various immunosuppressive agents currently available have a variety of actions. Methotrexate inhibits diverse cell types and functions, especially neutrophils and responses to inflammatory mediators, but may inhibit B lymphocyte differentiation. ${ }^{9}$ Azathioprine reduces the numbers of both T and B lymphocytes, and the precise action of chlorambucil is poorly understood. ${ }^{9}$ Cyclosporin $\mathrm{A}$ has a complex action but the principal immunosuppressive effect is by inhibition of CD4 positive Th2 lymphocyte activation and the related release of IL-4 and IL-5, thus inhibiting clonal expansion of $\mathrm{T}$ cells without directly affecting $\mathrm{B}$ cells. $^{9}$

In view of the known predominance of $\mathrm{T}$ cells in LIP, and the need to minimise generalised immunosuppression in a patient already antibody deficient, we hypothesised that cyclosporin A may be more appropriate than methotrexate or azathioprine whilst still allowing a steroid sparing effect. This was particularly important in our patient who already had significant morbidity from corticosteroid induced osteoporosis. The relapse of LIP when the cyclosporin levels became undetectable and the subsequent improvement with an increased dosage of cyclosporin A alone seem to confirm the efficacy of this drug in our patient. Furthermore, this therapeutic effect seems to occur at a low serum cyclosporin concentration of $<100 \mathrm{ng} / \mathrm{ml}$, and hence at a level where cyclosporin A toxicity can be kept to a minimum.

We conclude that low dose cyclosporin A may be a useful aid in the suppression of LIP, particularly in patients where steroid treatment presents particular problems.

1 Fishback N, Koss M. Update on lymphoid interstitial pneumonitis.Curr Opin Pulm Med 1996;2:429-33.

2 Nicholson AG, Wotherspoon AC, Diss TC, et al. Reactive pulmonary lymphoid disorders. Histopathology 1995;26: $405-12$.

3 Koss MN. Pulmonary lymphoid disorders. Semin Diagn Pathol 1995;12:158-71.

4 Koss MN, Hochholzer L, Langloss JM, et al. Lymphoid interstitial pneumonia: clinicopathological and immunopathological findings in 18 cases. Pathology 1987;19:17885.

5 Kohler PF, Cook RD, Brown WR, et al. Common variable hypogammaglobulinaemia with T-cell nodular lymphoid interstitial pneumonitis and B-cell nodular lymphoid hyperplasia: different lymphocyte populations with a similar response to prednisolone therapy. $\mathcal{F}$ Allergy Clin Immunol 1982;70:299-305.

6 Liebow AA, Carrington CB. Diffuse pulmonary lymphoreticular infiltrations associated with dysproteinaemia. Med Clin North Am 1973;57:809.

7 Strimlan CV, Rosenow EC, Weiland LH, et al. Lymphocytic interstitial pneumonitis. Review of 13 cases. Ann Intern Med 1978;88:616-21.

8 Report of a WHO Scientific Group. Primary immunodeficiency diseases. Clin Exp Immunol 1997;109(Suppl 1): $1-28$.

9 Lynch JP, McCune WJ. Immunosuppressive and cytotoxic pharmacology for pulmonary disorders. Am F Respir Crit Care Med 1997;155:395-420. 